\title{
The role of Nanog expression in tamoxifen- resistant breast cancer cells
}

This article was published in the following Dove Press journal:

OncoTargets and Therapy

3 June 2015

Number of times this article has been viewed

\author{
Khalid Arif ${ }^{\prime}$ \\ Issam Hussain' \\ Carol Rea' \\ Mohamed El-Sheemy ${ }^{2}$ \\ 'School of Life Sciences, University \\ of Lincoln, Brayford Pool, ${ }^{2}$ Lincoln \\ County Hospital, Greetwell Road, \\ Lincoln, Lincolnshire, UK
}

Correspondence: Khalid Arif

School of Life Sciences, University of Lincoln, Brayford Pool, Lincoln, LN6 7TS, UK

Email karif@lincoln.ac.uk

\begin{abstract}
There is an accumulation of evidence that shows a significant role of cancer stem cells in tumor initiation, proliferation, relapse, and metastasis. Nanog is the most important core transcription marker of stem cells, known by its role in maintaining pluripotency, proliferation, and differentiation. Therefore, this study aimed to examine the role of Nanog in breast cancer cell tamoxifen resistance and its implications in breast cancer treatment. In this study, the expression of the three core transcription markers Nanog, Oct3/4, and Sox 2 were quantitatively evaluated using flow cytometry. Then, small interfering RNA (siRNA) against human Nanog was transfected into tamoxifen-resistant breast cancer cells via Lipofectamine 2000. Nanog gene expression in the cells was detected using reverse transcription polymerase chain reaction (RTPCR). The change in cell proliferation was evaluated using the tetrazolium bromide method. An enzyme-linked immunosorbent assay was used to detect apoptosis of the transfected cells alone and in combination with 4-hydroxytamoxifen. The results showed a high level expression of Nanog, Oct3/4, and Sox 2 in MDA-MB-231 and MCF7/tamoxifen resistant cells compared with MCF7/wild-type. siRNA-mediated Nanog gene silencing can efficiently inhibit cell proliferation and induce apoptosis of tamoxifen-resistant breast cancer cells. This study provides a basis for further study of the role of Nanog in developing resistance to tamoxifen, its implication in breast cancer management, and as a new strategy to enhance response to endocrine therapy.
\end{abstract}

Keywords: breast cancer, cancer stem cell, Nanog, tamoxifen, estrogen receptor

\section{Introduction}

Breast cancer is the most commonly diagnosed cancer and the leading cause of cancer death in women worldwide. ${ }^{1}$ In the UK, the risk of developing breast cancer is 1 in 8 during a female's lifetime. ${ }^{2}$ Tamoxifen is the most frequently prescribed drug for treatment of estrogen receptor positive $\left(\mathrm{ER}^{+}\right)$breast cancer and to prevent the development of breast cancer in women with high risk of developing this disease. ${ }^{3}$ Approximately $70 \%$ of breast tumors are $\mathrm{ER}^{+}$; almost $50 \%$ of those either fail to respond or to initially respond to tamoxifen and eventually become resistant to treatment. ${ }^{4}$ Resistance to tamoxifen is a major clinical challenge in the treatment of breast cancer; however, the potential mechanisms of tamoxifen resistance are not fully understood but involve the coregulation crosstalk of ER and other signaling pathway networks. ${ }^{5}$ There is a growing body of evidence to support the role of cancer stem cells in tumorigenesis. ${ }^{6}$ All this evidence and these observations in relation to tumor cells and normal stem cells have been considered in the cancer stem cell hypotheses, which present a good model of self-renewal and pluripotentiality. ${ }^{7}$ 
Studies have shown that small subpopulations of cells are present in some tumors such as breast, colon, brain, pancreas, prostate, and some other tumor types. This cell fraction is defined as cancer stem cells (CSCs), which are characterized by their ability to self-renew, to initiate tumors, and to resist chemo/radiotherapy. ${ }^{8}$ Al-Hajj et al have shown that a small number of cells with $\mathrm{CD} 44^{+}$and CD24-/low lineage have a high ability to initiate a tumor when injected into severe combined immunodeficiency (SCID) mice. ${ }^{9}$ On the other hand, there are shared phenotypes between stem cells and cancer stem cells regarding the high presence of stemness gene markers such as Nanog, Sox2, and Oct3/4, which are found to be associated with poorly differentiated tumors and with high-grade ER ${ }^{-}$tumors. ${ }^{10}$ Liu et al reported that the MCF7/ Tmx (tamoxifen transfected) breast cancer cells induce CSC properties and may be responsible for tamoxifen resistance during breast cancer therapy. ${ }^{11}$ All this accumulative evidence in respect to the links between cancer and stem cells has important therapeutic implications. Nanog plays a crucial role in sustaining pluripotency in embryonic stem cells (ESCs) and promoting self-renewal. It was found to be expressed highly in some human tumors such as germ cell tumors and breast, cervix, oral cavity, renal, and ovarian carcinomas. ${ }^{12}$ However, the actual transcriptional mechanism of Nanog is still not clearly understood, but studies have established that epigenetic regulators promote Nanog transcription activity, whereas other studies indicated direct (positive or negative) transcription regulation, and a recent study stated that Nanog transcription has its own regulator (auto-regulator). ${ }^{13}$

The overexpression of Nanog has been used as a predictive factor for tumor progression in colorectal and oral cancer. ${ }^{12,14}$ In cervical cancer, the high expression of cytoplasmic Nanog was found to be associated with tumor growth, ${ }^{15}$ while a study by Chambers et al found an association between the downregulation of Nanog expression and an increase in differentiation tendency in ESCs. ${ }^{16}$ In breast cancer, Nanog gene expression was found to correlate directly with cell differentiation. ${ }^{10}$ Using the MCF7 breast cancer cell line, the blocking of Nanog inhibited tumor growth and expansion. ${ }^{12}$ Furthermore, data have shown a correlation between Nanog expression and stage and treatment prognosis in patients with cervical neoplasia. In addition, Nanog was found to support the stem-like and immune-resistant phenotype of tumor cells through the Akt pathway, which is involved in cell survival and apoptosis. Moreover, in the same study, Noh et al evaluated the therapeutic implications of Nanog via injecting a colorectal cancer cell line (HCT116/SCT-E7) into NOD/SCID mice, and then, knocking down the Nanog using chitosan nanoparticles, they found that the silencing of Nanog enhanced immune-based therapy. ${ }^{17}$ Hence, a study by Shan et al in 2012 of hepatocellular carcinoma indicated that the loss of Nanog eliminated the self-renewal ability of the human hepatocellular carcinoma cell line, whereas the overexpression of Nanog restored this ability. ${ }^{18}$ Yet, the role of Nanog in developing resistance to tamoxifen in breast cancer has not been investigated until now. In particular, studies have not investigated whether Nanog could be a therapeutic target in breast cancer endocrine therapy resistance.

Therefore, the aim of this study was to investigate the expression of the transcription factors (Nanog, Oct3/4, and Sox2) in tamoxifen-resistant breast cancer, provide evidence about the role of Nanog in development of tamoxifen resistance in breast cancer, and finally to enhance the response to tamoxifen.

\section{Materials and methods Cell lines and cell culture}

The ER- breast cancer cell line MDA-MB-231 was purchased from European Collection of Cell Cultures (ECACC), and MCF7/Tmx and parental MCF7/wild-type were kindly donated by Dr Saraswati Sukumar (Johns Hopkins University, Baltimore, MD, USA). All cells were routinely cultured in Dulbecco's Modified Eagle's Medium (DMEM) (Sigma-Aldrich); supplemented with $10 \%$ fetal calf serum (Sigma-Aldrich), 100 units/mL penicillin-streptomycin, and $2 \mathrm{mM}$ L-glutamine (Sigma-Aldrich) in a humidified atmosphere of $5 \% \mathrm{CO}_{2}$ in air at $37^{\circ} \mathrm{C}$; and used when the culture reached $85 \%-90 \%$ confluence.

\section{Tamoxifen cytotoxicity}

Cells were grown in complete DMEM for 48 hours. Then, 4-hydroxytamoxifen (4-OHT) (Sigma-Aldrich) was dissolved in ethanol and added to the culture media to produce a range of drug concentrations $(1-80 \mu \mathrm{M})$, and the cells were incubated with the drug for an additional 48 hours. Ethanol concentrations added with 4-OHT to the culture media were less than $0.3 \%$ and had no effect on cell growth (viability tested). ED50 values were calculated for each of the two tamoxifen-resistant breast cancer cell lines and the tamoxifen responsive cell line. Tamoxifen effects were also tested in separate experiments in the small interfering RNA (siRNA) Nanog-transfected cells to evaluate the growth rate and detect the apoptosis. Vehicle treated cells $(0.5 \%$ 
$\mathrm{v} / \mathrm{v}$ ethanol) were always included and were not found to affect the results.

\section{Flow cytometric analysis}

To measure Nanog, Oct4, and Sox2, cells were fixed in $1 \mathrm{~mL}$ ice cold methanol (Thermo Fisher Scientific) for 10 minutes at $-20^{\circ} \mathrm{C}$ and washed twice in phosphate-buffered saline (PBS) $1 \%$ bovine serum albumin (BSA) (Sigma-Aldrich). Cells were then incubated in permeabilization buffer $0.3 \%$ Triton X-100 in $1 \%$ BSA (Sigma-Aldrich) at room temperature for 45 minutes. Cells were labeled with human Nanog phycoerythrin polyclonal antibody goat IgG, human Oct4 MAb mouse IgG and human Sox 2 monoclonal antibody mouse IgG (R\&D System), diluted in permeablization solution at a final concentration according to the manufacturer's recommendation and incubated for 30 minutes at $2^{\circ} \mathrm{C}-8^{\circ} \mathrm{C}$. Cells were washed three times by centrifugation at $400 \times g$ for 5 minutes and resuspended in ice cold PBS. Cells were labeled with appropriate secondary antibody diluted in $3 \% \mathrm{BSA} / \mathrm{PBS}$ for 30 minutes at $2^{\circ} \mathrm{C}-8^{\circ} \mathrm{C}$ in the dark. Cells were washed and resuspended in ice cold PBS and 3\% BSA and immediately analyzed on Epics xL flow cytometer (Beckman Coulter, High Wycombe, UK).

\section{siRNA transfection}

Nanog siRNA was obtained from Life Technologies, UK. Breast cancer cell lines were transfected with siRNA at concentrations of 1-10 $\mathrm{nM}$ using $0.1 \%$ (v/v) Lipofectamine RNAiMAX (Life Technologies) according to the manufacturer's instructions. Cell media was changed to serum-containing media 24 hours after transfection, and cells were maintained in regular culture medium for 48-72 hours before analysis. Additionally, for each cell line, the siRNA was cotransfected into the cells with reporter plasmids to assess the efficiency of Nanog knockdown using TYE 563M (Origen Biomedical, Austin, TX, USA) labeled transfection control siRNA for each of the transfected cell lines and examined using fluorescence microscopy (Nikon, Kingston upon Thames, UK).

\section{RT-PCR analysis for siRNA quantification}

Reverse transcription polymerase chain reaction (RT-PCR) through cyclical amplification was performed to enable evaluation of Nanog gene expression prior to and after gene silencing. Total RNA was extracted from breast cancer cell lines using the RNeasy kit (Life Technologies). At 48 hours posttransfection, $1 \mu \mathrm{g}$ of total RNA was used to synthesize cDNA using cDNA kit (Life Technologies) as per manufac- turer's instructions. TaqMan gene expression assay kit (Life Technologies) reactions were performed using the ABI 7000 real-time PCR machine, and the experimental cycle threshold was calibrated against that of the standard.

\section{Transfected cell growth rate}

Cells were seeded and transfected in a 96-well plate; cell growth continued 48 hours from transfection in complete media. The wells were then treated with $50 \mu \mathrm{L}$ of $0.1 \mathrm{mg} / \mathrm{mL}$ tetrazolium bromide (MTT) (Sigma-Aldrich), the absorbance was measured at a wavelength of $570 \mathrm{~nm}$ using a microplate reader (BMG Labtech, Aylesbury, UK), and the growth curves were plotted for each cell line.

\section{Determination of the apoptosis markers ( $\mathrm{Bcl}-2$ and annexin $\mathrm{V}$ ) by enzyme-linked immunosorbent assay (ELISA)}

Briefly, Nanog siRNA transfected MDA-MB-231, MCF7/ Tmx, and parental MCF7/WT cells were incubated with 4-OHT overnight. Cell lysates were prepared, and the level of Bcl-2 and annexin $\mathrm{V}$ were determined for the treated and untreated (control) cells using Bcl-2 and annexin V ELISA.

\section{Analysis of $\mathrm{Bcl}-2$ using the quantitative ELISA}

The human Bcl-2 ELISA kit (eBioscience, Vienna, Austria) was used to quantitatively analyze Bcl-2 protein prior to and after siRNA transfection in response to 4-OHT. Cells were washed twice with PBS and resuspended with lysis buffer for 60 minutes at room temperature. Cells were recentrifuged and resuspended in sample diluent at a ratio of 1:5. Duplication of each cell lysate sample and a set of standards in duplicate containing serially-diluted (1:2) of lyophilized Bcl-2 standards. Biotin-conjugate anti human Bcl-2 monoclonal antibody diluted 1:100 in the assay buffer were added to each ELISA well and incubated for 2 hours at room temperature. Then the assay was completed as per manufacturer's instructions. The absorbance of each microwell was then read at $450 \mathrm{~nm}$ using an ELISA plate reader (BMG LABTECH Ltd., Aylesbury, UK).

\section{Analysis of annexin $\mathrm{V}$ using the quantitative ELISA} Annexin V ELISA kit was purchased from Abnova, Taipei City, Taiwan. Standard and negative controls were washed thoroughly twice with $400 \mu \mathrm{L}$ of wash buffer per well. The standard dilution and samples were prepared and added to the allocated microwells as per manufacturer's instructions. Absorbance was measured at $450 \mathrm{~nm}$ using a plate reader (BMG Lab Technologies). 
A
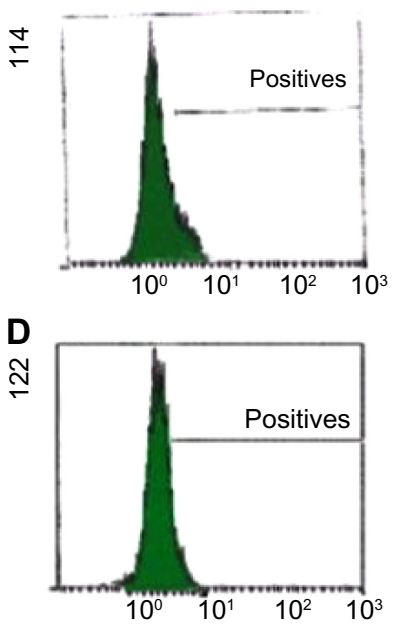

G

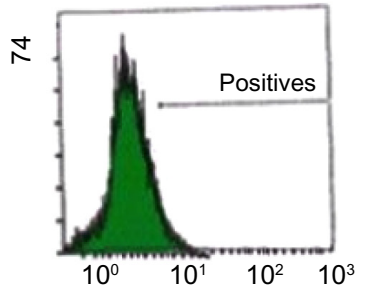

B

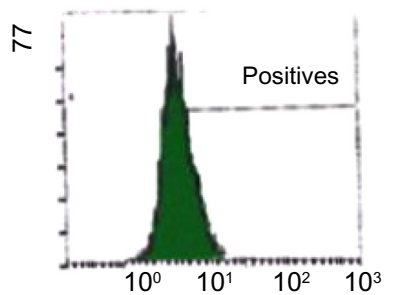

E

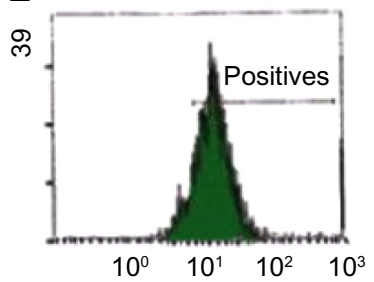

H

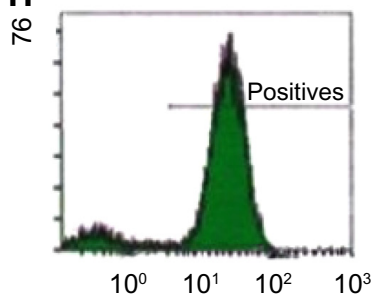

C

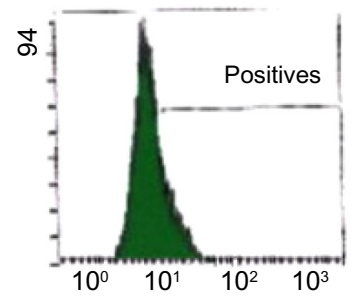

F

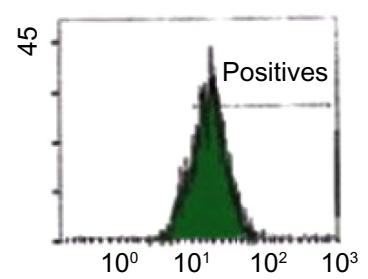

I

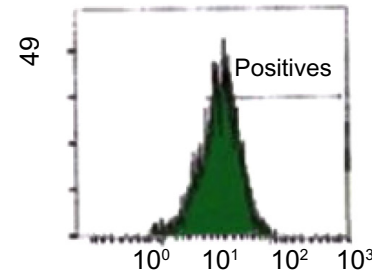

Figure I Flow cytometric analysis of intracellular staining of embryonic markers (Nanog, Oct4, and Sox2) in breast cancer cells (MCF7/WT, MDA-MB-23I, and MCF7/Tmx). Notes: $\left(\right.$ A-C) cells were labeled using anti-mouse Nanog Alexa Fluor ${ }^{\circledR} 488$ antibody, showing 12\%, 68\%, and 49\% positivity in MCF7/WT, MDA-MB-23I, and MCF7/Tmx, respectively. (D-F) cells were labeled using goat anti-human Oct4, followed by anti-goat IgG secondary antibody showing I4\%, 52\%, and 55\% positivity in MCF7/WT, MDA-MB-23 I and MCF7/Tmx, respectively. (G-I) cells were labeled using human/mouse Sox 2 monoclonal antibody, followed by anti-mouse lgG secondary antibody, showing II\%, $70 \%$, and $59 \%$ positivity in MCF7/NT, MDA-MB-23I, and MCF7/Tmx, respectively. All the data represent the mean value from three independent experiments ( $\mathrm{n}=3$ ) \pm SE.

Abbreviations: SE, standard error; WT, wild type; Tmx, tamoxifen transfected.

\section{Statistical analysis}

Data are expressed as the mean \pm standard error (SE). Statistically significant differences were determined using one-way or two-way analysis of variance, SPSS version 21 software (IBM). $P<0.05$ was considered to indicate a statistically significant result.

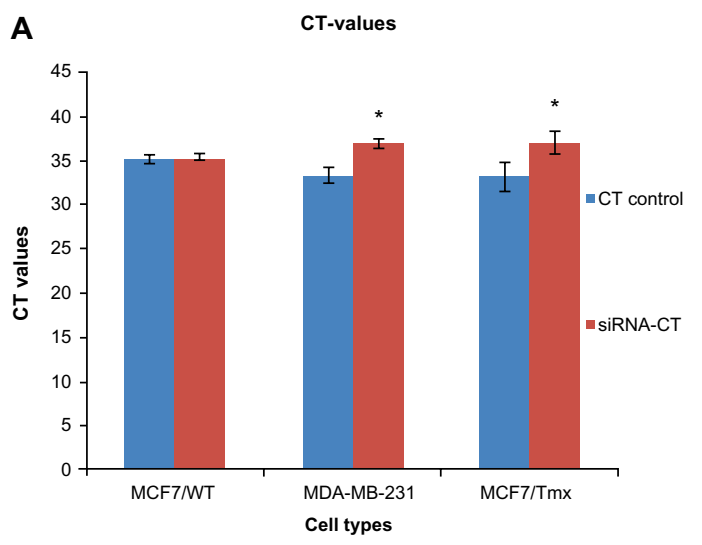

\section{Results}

\section{Cytotoxic effects of tamoxifen}

The exposure of cells to 4-OHT induced growth inhibitory effects with median ED50 values of $17.5 \mu \mathrm{M}, 25 \mu \mathrm{M}$, and $32 \mu \mathrm{M}$ for MCF7/WT, MCF7/Tmx, and MDA-MB-231 respectively.

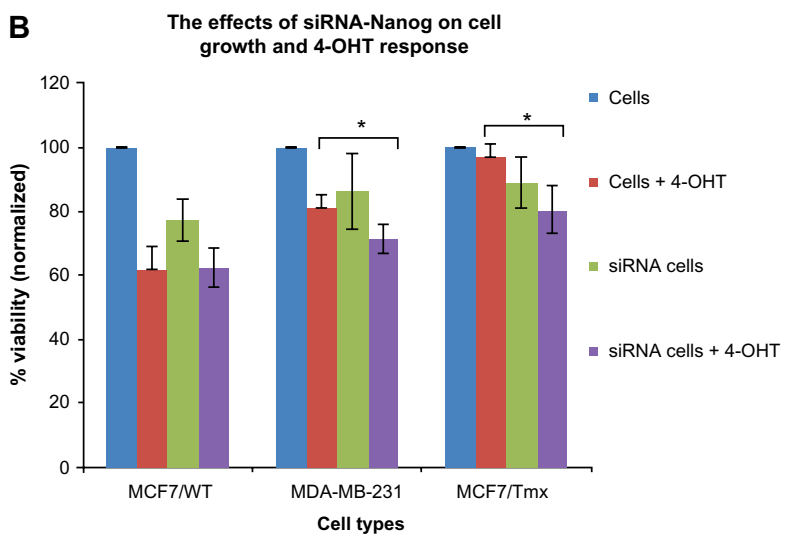

Figure 2 CT representing the RT-PCR amplifications and the effect of Nanog silencing (siRNA) on the cell growth.

Notes: (A) Results of qPCR are expressed as the CT (cycle of threshold) value for each transfected and nontransfected cell line. Data represent the mean value from two independent experiments $\pm S E$. $* P<0.05$ indicates significant difference between transfected and nontransfected cell lines. (B) The effects of Nanog silencing (siRNA) on the growth of the cells and response to tamoxifen. Results were normalized to viability percentages. The results are an average of three, in duplicate independent experiments ( $\mathrm{n}=3$ ).

Abbreviations: qPCR, quantitative polymerase chain reaction; SE, standard error; siRNA, small interfering RNA; 4-OHT, 4-hydroxytamoxifen; WT, wild-type; Tmx, tamoxifen transfected; RT-PCR, reverse transcription polymerase chain reaction. 

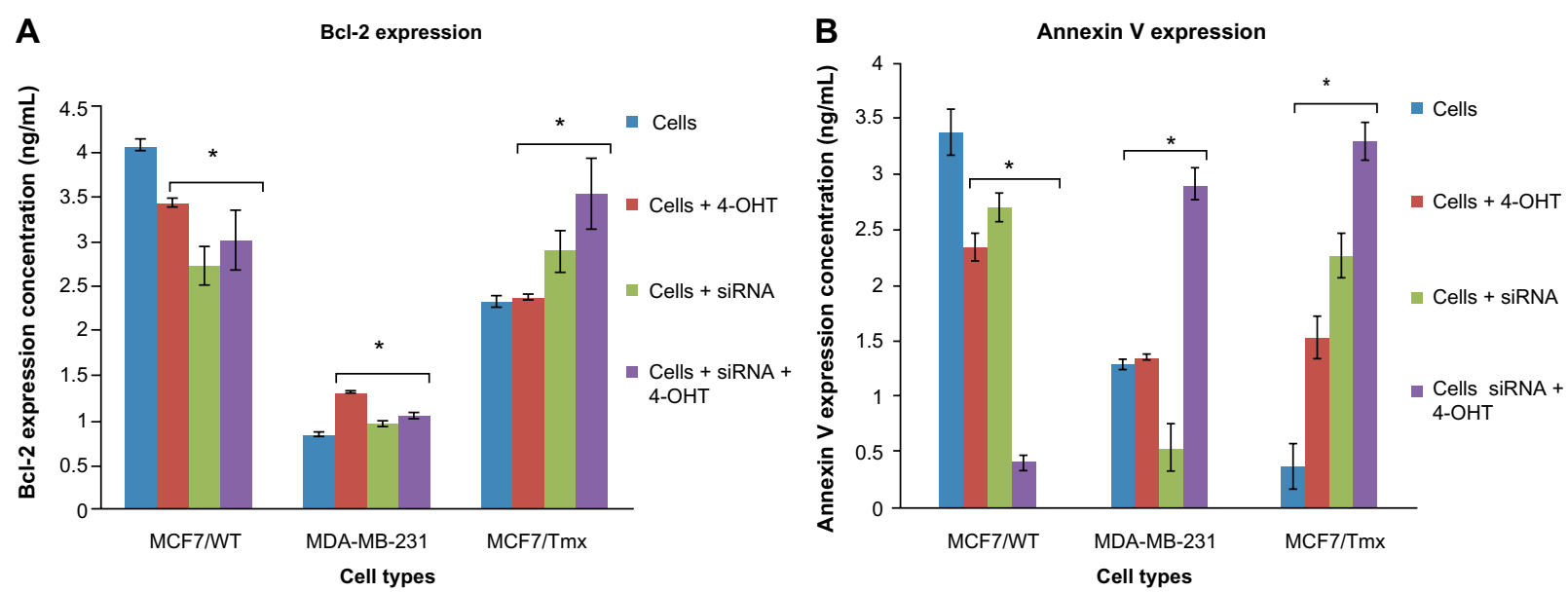

Figure 3 The expression of $\mathrm{Bcl}-2$ and Annexin $\mathrm{V}$, data are representative of three independent experiments.

Notes: (A) Expression of Bcl-2 (ng/mL) in three Nanog knocked down (siRNA) breast cancer cell lines. The expression was evaluated using human Bcl-2 ELISA in three independent experiments and in duplicate for each experiment. Results are expressed as the mean \pm SE. Statistical analysis was performed by one-way ANOVA using SPSS software. $* P<0.05$ compared with control. (B) Expression of annexin $V$ concentration $(\mathrm{ng} / \mathrm{mL}$ ) in Nanog knocked down (siRNA) of the three breast cancer cell lines. The expression was evaluated using human annexin $\vee$ ELISA in three independent experiments and in duplicate for each experiment \pm SE. $* P<0.05$ compared with control. Abbreviations: siRNA, small interfering RNA; ELISA, enzyme-linked immunosorbent assay; ANOVA, analysis of variance; SE, standard error; 4-OHT, 4-hydroxytamoxifen; WT, wild-type; Tmx, tamoxifen transfected.

\section{Stemness transcription factor}

Stemness protein levels were examined using flow cytometry. As shown in Figure 1, positive expression of Nanog, Oct4, and Sox 2 were observed in tamoxifen-resistant cells MCF7/ Tmx and MDA-MB-231 compared with low expression of these factors in parental MCF7/WT.

\section{Nanog knockdown inhibits cell proliferation and induces apoptosis}

Quantitative PCR (qPCR) through cyclical amplification was performed to enable quantification of Nanog gene expression prior to and after siRNA treatment. The experimental cycle threshold was calibrated against that of standard and all amplifications were performed in duplicate (Figure 2A). The effect of Nanog knockdown in combination with 4-OHT treatment on cells was assessed after monitoring for 48 hours (Figure 2B). Data represent mean \pm SE of three independent experiments $(P<0.05)$; 4-OHT significantly inhibits growth in MCF7/Tmx and MDA-MB-231 in comparison with nontransfected cells. Quantitative evaluation of Bcl-2 expression was performed using ELISA prior to and after the knockdown of Nanog was carried out. Low expression of Bcl-2 was observed in the $\mathrm{ER}^{-}$breast cancer cell line MDA-MB-231, and this expression increased significantly in response to 4-OHT alone $(P<0.05)$ (Figure $3 \mathrm{~A})$. In the same way, the siRNA transfected MDA-MB-231cell line expressed a slightly higher but not significant Bcl-2 level $(P>0.05)$ when cells were incubated with 4-OHT. Whereas a high expression of $\mathrm{Bcl}-2$ was indicated in the control MCF7/WT cell line and this expression reduced after the silencing of the Nanog gene and in response to 4-OHT. In contrast, the expression of Bcl-2 in the MCF7/Tmx breast cancer cell line was increased significantly after silencing of the Nanog gene $(P<0.05)$. The evaluation of annexin $\mathrm{V}$ expression was performed by means of ELISA prior to and after the knockdown of Nanog. A high expression of annexin $\mathrm{V}$ was indicated in the tamoxifen responsive cell line MCF7/WT, and this expression reduced significantly $(P<0.05)$ when the siRNA transfected cells were incubated with 4-OHT (Figure 3B). In contrast to the parental MCF7/WT, the MCF7/Tmx and MDA-MB-231 cell lines showed a high expression of annexin $\mathrm{V}$ when the transfected cells were incubated with 4-OHT $(P<0.05)$. This expression was significantly lower in the control MCF7/Tmx and in the non-tamoxifen treated transfected MDA-MB-231 cells.

\section{Discussion and conclusion}

Development of resistance to tamoxifen remains an important clinical problem. In this study, high expression of embryonic markers (Oct4, Sox2, and Nanog) were indicated in ER ${ }^{-}$MDA-MB-231 and MCF7/Tmx, and these expressions were inversely correlated with ER expression and response to tamoxifen (Figure 1). In addition, the current study showed the key role of Nanog in the development of tamoxifen resistance and that reduction in the endogenous level of Nanog decreases the proliferation rate and induces tamoxifen sensitivity.

A correlation between embryogenesis and oncogenesis has become a main subject in the study of cancer biology 
and therapies, which require the existence of stem/progenitor cells that are regulated in response to systemic and local signaling. ${ }^{19,20}$ Previous studies showed the role of cancer stem cells in developing the resistance to therapeutic agents and tumor initiation. Based on CD24-/low/CD $44^{+}$surface marker expression, a population of cancer stem cells has been isolated, characterized by their ability to form tumors in SCID mice, even when injected with as few as 200 cells. ${ }^{9}$ In addition, the survival of the CD24-/low/CD44+ cancer-initiating cells that have been isolated from MCF7 and MDA-MB-231 breast cancer cells in response to radiation, suggests the role of stem/progenitor cells in resistance to therapeutic agents and enhancing survival mechanisms. ${ }^{21}$ Furthermore, there are other studies that supported the recent results when the response of the subpopulation with $\mathrm{CD} 24^{-} /$low/CD $44^{+}$to chemotherapy was evaluated; these studies found that the chemotherapy treatment increases cancer stem cell properties after the treatment. ${ }^{22}$ In line with their findings, our data further showed that the expression levels of Oct4, Sox2, and Nanog might be simultaneously or individually correlated with response to tamoxifen as in MDA-MB-231 and MCF7/ Tmx in comparison with MCF7/WT (Figure 2B).

Nanog has been identified as a transcription factor, and its role in maintain self-renewal in ESCs has been established in previous studies. Also high expression of Nanog has been found to be associated with high pluripotency in ESCs, and that Nanog promotes ESC differentiation. ${ }^{23}$ To know whether Nanog participates in the regulation of tamoxifen resistance in breast cancer, we performed a Nanog knockdown and then measured the growth rate along with apoptosis markers. Results revealed an important role of Nanog in regulating growth in breast cancer cells. The blocking of Nanog using siRNA showed a significant arrest in the growth of the tamoxifen-resistant cells, as shown in Figure 2B. This is in agreement with a previous study in hepatocellular carcinoma. ${ }^{18}$ However, there is not a clear mechanism to explain the relation between the expression of ER and the stemness genes, but CSCs are known to have a lack of ER expression; at the same time tamoxifen action is dependent on the function of expressed ER. ${ }^{24,25}$ Therefore, further investigation was carried out to evaluate cellular apoptosis. Enhancements of apoptosis were indicated after blocking of Nanog, suggesting that Nanog might play a relevant role in enhancing tamoxifen action, which could be via an alternative mechanism, not only via ER dependent mechanisms. ${ }^{25}$

Knowing that the Bcl-2 family plays an important regulatory role in apoptosis, either as activator (Bax) or as inhibitor (Bcl-2), interestingly, in this study, the MCF7/WT expressed a high level of Bcl-2 in comparison with both resistant (MCF7/
Tmx and MDA-MB-231) cell lines (Figure 3A). This is in agreement with a previous clinical study published by Daidone et $\mathrm{al},{ }^{26}$ who showed an association between the overexpression of Bcl-2 and ER expression along with other biologic features of a differentiated phenotype such as slow proliferation, progesterone receptor expression, and human epidermal growth factor receptor 2 (Her2) expression. Also, this study found that women with $\mathrm{ER}^{+}$and $\mathrm{Bcl}-2^{-}$breast cancer have worse prognoses than those with $\mathrm{ER}^{-}$and $\mathrm{Bcl}-2^{+}$breast cancer. ${ }^{26}$ Furthermore, the high expression of $\mathrm{Bcl}-2$ could be related to the lack of caspase 3 expression. ${ }^{27}$ As it is not clear why Bcl-2 represents a molecular marker for better prognosis in breast cancer, the cell death was quantified using annexin V ELISA to determine the real implication of the siRNA on response to tamoxifen (Figure 3B). A low expression of annexin $\mathrm{V}$ was indicated in the tamoxifen-resistant cell line, and this expression increased when cells were treated with a combination of siRNA Nanog and 4-OHT, indicating an enhancement of apoptosis.

A previous study of tamoxifen cytotoxicity showed that annexin $\mathrm{V}$ expression in response to tamoxifen is time- and dose-dependent, that there is a peak value, and that soon after that, the expression reduces sharply in ER- breast cancer cells. This might explain the significant expression of annexin $\mathrm{V}$ in MDA-MB-231 breast cancer cells when treated with 4-OHT and the lack of a significant increase in expression of annexin $\mathrm{V}$ in MCF7/WT. This atypical expression of annexin $\mathrm{V}$ as reported in a previous study is mainly caused by the irregular expression of associated caspase 3 in response to tamoxifen. ${ }^{28}$ However, that may not be a full and accurate explanation of the low expression of annexin $\mathrm{V}$ in siRNA MDA-MB-231 cells or in siRNA and 4-OHT treatment of MCF7/WT and could be related to the deregulation of Nanog and its effect on the apoptosis and cell cycle arrest. Further study is needed to find out the effects of the stemness gene in the regulation of apoptosis and how that reflects on the presence and absence of caspase 3, 8, and 9. In addition, further studies could include DNA fragmentation to indicate early and late apoptosis and secondary necrosis.

In addition, another study showed that tamoxifen could mediate its apoptosis effects via activation of the caspase 3 pathway in $\mathrm{ER}^{-}$breast cancer cells. ${ }^{29}$ These previous studies in both $\mathrm{ER}^{+}$and $\mathrm{ER}^{-}$breast cancer cells are in agreement with the finding in the study reported here. At the same time, the expression of Bcl-2 increased in both resistant cell lines (Figure 3A), which is in agreement with previous studies that identified an association between the expression of Bcl-2 and ER status, with improved survival from breast cancer. ${ }^{30}$ Also, 
it has been reported that $\mathrm{ER} \alpha$ influenced the survival of breast cancer cells through regulation of Bcl-2/Bax. ${ }^{31}$

In a parallel experiment, quantitative expression of annexin $\mathrm{V}$ was determined by ELISA after 48 hours of interference with siRNA Nanog. As shown in Figure 3B, the combination of siRNA Nanog and 4-OHT caused an increase in expression of annexin V in MDA-MB-231 and MCF7/Tmx cells. In contrast, this expression was reduced in $\mathrm{ER}^{+} \mathrm{MCF}-7$ cells significantly. The results confirmed that the blocking of Nanog was very effective in inducing apoptosis in ER ${ }^{-}$MDA-MB-231 and MCF7/Tmx cells, whereas it had no effect in MCF7/WT cells.

However, the mechanism of tamoxifen resistance is still not fully understood, but it is known that tamoxifen's effect depends on the expression of ER; mainly, the development of tamoxifen resistance is associated with a loss of ER. ${ }^{32}$ The current results showed that the lack of ER in the MDA-MB-231 cell line was correlated with positive core transcription factor expression. This correlation was still significant in the acquired tamoxifen-resistant cell line MCF7/Tmx in comparison with the parental MCF7/WT. These results might indicate an inverse correlation between the cancer stem cells and the expression of ER, which might explain the resistance to tamoxifen. ${ }^{24,33}$ The results from this study indicated that the role of Nanog is not limited to the development of tumorigenesis only, but also plays a role in the development of resistance to tamoxifen, and it is inversely correlated with ER expression and apoptosis. The implication of this finding is that a combination of tamoxifen and Nanog interference could be developed as a new treatment to enhance response to tamoxifen and to induce apoptosis.

However, the expression of annexin $\mathrm{V}$ was not detected in MCF7 cells in response to siRNA and 4-OHT; this suggests that in MCF7 cells there may be another mechanism of apoptosis, which could be a caspase 3 independent pathway. There are many studies dealing with apoptosis in MCF7 in response to different therapeutic agents. All studies show DNA fragmentation despite the lack of caspase $3,{ }^{34}$ or apoptosis could be because of the effect of tamoxifen on Bcl-2 expression. A study by Zhang et al showed that the downregulation of Bcl-2 correlated with tamoxifen-induced apoptosis, and this is in agreement with the current results of the expression of $\mathrm{Bcl}-2$ and annexin $\mathrm{V}$ in MCF7/WT. ${ }^{35}$ However, any findings different from the current results could be due to the differences in the variants of MCF7 cells used, ${ }^{36}$ because the results for the Bcl-2 and annexin $\mathrm{V}$ expression showed a clear significant effect of the siRNA Nanog on the expression of the Bcl-2 and apoptosis (annexin V) in MDA-MB-231 and MCF7/ Tmx breast cancer cells. Further study of DNA fragmentation and the effects of Nanog on caspase 3, 6, 7, and 9 expression will be useful.

The results from this study indicated that the role of Nanog is not limited to the development of tumorigenesis only, but Nanog also has a role in the development of resistance to tamoxifen, and it is inversely correlated with ER expression and the apoptosis pathway. The results are in agreement with a recent study published by Piva et al that shows that the silencing of the Sox 2 gene in ER- breast cancer reduced the size of the stem/progenitor cell population and restored response to tamoxifen treatment. ${ }^{37}$

The implication of this finding is that a combination of tamoxifen and Nanog interference could be developed as new treatment to enhance the response to tamoxifen and to induce apoptosis.

\section{Disclosure}

The authors report no conflicts of interest in this work.

\section{References}

1. Jemal A, Bray F, Center MM, Ferlay J, Ward E, Forman D. Global cancer statistics. CA Cancer J Clin. 2011;61(2):69-90.

2. Cancerresearchuk.org [homepage on the internet]. Cancer Research UK; 2013 [updated April 25, 2013; cited February 9, 2014]. Available from: http://www.cancerresearchuk.org/cancer-info/cancerstats/types/breast/ incidence/uk-breast-cancer-incidence-statistics. Accessed May 10, 2014.

3. Schiff R, Massarweh S, Shou J, Osborne CK. Breast cancer endocrine resistance how growth factor signaling and estrogen receptor coregulators modulate response. Clin Cancer Res. 2003;9(1):447S-454S.

4. García-Becerra R, Santos N, Díaz L, Camacho J. Mechanisms of resistance to endocrine therapy in breast cancer: focus on signaling pathways, miRNAs and genetically based resistance. Int J Mol Sci. 2013;14:108-145.

5. Thrane S, Lykkesfeldt AE, Larsen MS, Sorensen BS, Yde CW. Estrogen receptor $\alpha$ is the major driving factor for growth in tamoxifen-resistant breast cancer and supported by HER/ERK signaling. Breast Cancer Res Treat. 2013;139(1):71-80.

6. Diehn M, Cho RW, Lobo NA, et al. Association of reactive oxygen species levels and radioresistance in cancer stem cells. Nature. 2009; 458(7239):780-783.

7. Visvader JE, Lindeman GJ. Cancer stem cells in solid tumours: accumulating evidence and unresolved questions. Nat Rev Cancer. 2008;8(10):755-768.

8. Vezzoni L, Permian G. Limitations of the cancer stem cell theory. Cytotechnology. 2008;58(1):3-9.

9. Al-Hajj M, Wicha MS, Benito-Hernandez A, Morrison SJ, Clarke MF Prospective identification of tumorigenic breast cancer cells. Proc Natl Acad Sci U S A. 2003;100(7):3983-3988.

10. Ben-Porath I, Thomson MW, Carey VJ, et al. An embryonic stem cell-like gene expression signature in poorly differentiated aggressive human tumors. Nat Genet. 2008;40:499-507.

11. Liu H, Zhang HW, Sun XF, et al. Tamoxifen-resistant breast cancer cells possess cancer stem-like cell properties. Chin Med J (Engl). 2013;126(16):3030-3034. 
12. Jeter CR, Badeaux M, Choy G, et al. Functional evidence that the selfrenewal gene NANOG regulates human tumor development. Stem Cells. 2009;27(5):993-1005.

13. Fidalgo M, Faiola F, Pereira CF, et al. Zfp281 mediates Nanog autorepression through recruitment of the NuRD complex and inhibits somatic cell reprogramming. Proc Natl Acad Sci U S A. 2012;109(40): $16202-16207$

14. Meng HM, Zheng P, Wang XY, et al. Overexpression of nanog predicts tumor progression and poor prognosis in colorectal cancer. Cancer Biol Ther. 2010;9(4):295-302.

15. Gu TT, Liu SY, Zheng PS. Cytoplasmic NANOG-positive stromal cells promote human cervical cancer progression. Am J Pathol. 2012;181(2):652-661.

16. Chambers I, Silva J, Colby D, et al. Nanog safeguards pluripotency and mediates germline development. Nature. 2007;450(7173): $1230-1234$

17. Noh KH, Kim BW, Song KH, et al. Nanog signaling in cancer promotes stem-like phenotype and immune evasion. $J$ Clin Invest. 2012;122(11):4077-4093.

18. Shan J, Shen J, Liu L, et al. Nanog regulates self-renewal of cancer stem cells through the insulin-like growth factor pathway in human hepatocellular carcinoma. Hepatology. 2012;56(3):1004-1014.

19. Croce CM. Oncogenes and cancer. $N$ Engl J Med. 2008;358(5): 502-511.

20. Brabletz T. To differentiate or not - routes towards metastasis. Nat Rev Cancer. 2012;12(6):425-436.

21. Phillips TM, McBride WH, Pajonk F. The response of CD24-/low/ CD44+ breast cancer-initiating cells to radiation. J Natl Cancer Inst. 2006;98(24):1777-1785.

22. Li X, Lewis MT, Huang J, et al. Intrinsic resistance of tumorigenic breast cancer cells to chemotherapy. J Natl Cancer Inst. 2008;100(9): $672-679$.

23. Ling GQ, Chen DB, Wang BQ, Zhang LS. Expression of the pluripotency markers Oct3/4, Nanog and Sox 2 in human breast cancer cell lines. Oncol Lett. 2012;4:1264-1268.

24. Clayton H, Titley I, Vivanco Md. Growth and differentiation of progenitor/stem cells derived from the human mammary gland. Exp Cell Res. 2004;297(2):444-460.

25. Ring A, Dowsett M. Mechanisms of tamoxifen resistance. Endocr Relat Cancer. 2004;11(4):643-658.
26. Daidone MG, Luisi A, Veneroni S, Benini E, Silvestrini R. Clinical studies of Bcl-2 and treatment benefit in breast cancer patients. Endocr Relat Cancer. 1999;6(1):61-68.

27. Liang Y, Yan C, Schor NF. Apoptosis in the absence of caspase 3. Oncogene. 2001;20(45):6570-6578.

28. Salami S, Karami-Tehrani F. Biochemical studies of apoptosis induced by tamoxifen in estrogen receptor positive and negative breast cancer cell lines. Clin Biochem. 2003;36(4):247-253.

29. Mandlekar S, Yu R, Tan TH, Kong AN. Activation of caspase-3 and c-Jun NH2-terminal kinase-1 signalling pathways in tamoxifen-induced apoptosis of human breast cancer cells. Cancer Res. 2000;60(21): 5995-6000.

30. Dawson SJ, Makretsov N, Blows FM, et al. BCL2 in breast cancer: a favourable prognostic marker across molecular subtypes and independent of adjuvant therapy received. Br J Cancer. 2010;103(5): 668-675.

31. Yang Q, Sakurai T, Jing X, et al. Expression of Bcl-2, but not Bax, correlates with estrogen receptor status and tumor proliferation in invasive breast carcinoma. Pathol Int. 1999;49(9):775-780.

32. García-Becerra R, Santos N, Díaz L, Camacho J. Mechanisms of resistance to endocrine therapy in breast cancer: focus on signalling pathways, miRNAs and genetically based resistance. Int J Mol Sci. 2012;14(1):108-145.

33. Liu W, Swetzig WM, Medisetty R, Das GM. Estrogen-mediated upregulation of Noxa is associated with cell cycle progression in estrogen receptor-positive breast cancer cells. PloS One. 2011;6(12):e29466.

34. Kumar R, Vadlamudi RK, Adam L. Apoptosis in mammary gland and cancer. Endocr Relat Cancer. 2000;7(4):257-269.

35. Zhang GJ, Kimijima I, Onda M, et al. Tamoxifen-induced apoptosis in breast cancer cells relates to down-regulation of bcl-2, but not bax and bcl-X(L), without alteration of p53 protein levels. Clin Cancer Res. 1999;5(10):2971-2977.

36. Osborne CK, Hobbs K, Trent JM. Biological differences among MCF-7 human breast cancer cell lines from different laboratories. Breast Cancer Res Treat. 1987;9(2):111-121.

37. Piva M, Domenici G, Iriondo O, et al. Sox 2 promotes tamoxifen resistance in breast cancer cells. EMBO Mol Med. 2014;6(1):66-79.
OncoTargets and Therapy

\section{Publish your work in this journal}

OncoTargets and Therapy is an international, peer-reviewed, open access journal focusing on the pathological basis of all cancers, potential targets for therapy and treatment protocols employed to improve the management of cancer patients. The journal also focuses on the impact of management programs and new therapeutic agents and protocols on

\section{Dovepress}

patient perspectives such as quality of life, adherence and satisfaction The manuscript management system is completely online and includes a very quick and fair peer-review system, which is all easy to use. Visit http://www.dovepress.com/testimonials.php to read real quotes from published authors. 